\title{
Unexpected remission of hyperparathyroidism caused by hemorrhage due to the use of fine-needle aspiration biopsy: two cases report
}

\author{
Joon Ho, Donggyu Kim, Ji-eun Lee, Soonmin Choi, Hyeryeon Choi, Sunhyung Choi, Jinkyung Kim, \\ Sang-Wook Kang, Jandee Lee, Jong Ju Jeong, Kee-Hyun Nam, Woong Youn Chung \\ Department of Surgery, Severance Hospital, Yonsei Cancer Center, Yonsei University College of Medicine, Seoul, Korea \\ Correspondence to: Jong Ju Jeong, MD, PhD. Department of Surgery, Yonsei University College of Medicine, Seoul 03722, Korea. \\ Email: JUNGJONGJ@yuhs.ac.
}

\begin{abstract}
Hyperparathyroidism is not a rare disease; if a parathyroid adenoma is confirmed, the treatment of choice is the surgical resection. Diagnostic use of fine-needle aspiration biopsy (FNAB) for histological confirmation in patients with hyperparathyroidism is controversial. And spontaneous remission of hyperparathyroidism caused by bleeding or infarction of the adenoma rarely occurs. Here we have reported two cases of hyperparathyroidism in which spontaneous remission occurred due to the use of FNAB for diagnosis. The remission was confirmed after surgical removal and pathological review of the adenoma. The first patient diagnosed with primary hyperparathyroidism (PHPT) had neck pain and severe swelling 4 days after FNAB, and spontaneous remission due to intracapsular hemorrhage was confirmed after surgery. In the second patient receiving hemodialysis treatment for end-stage renal disease, hyperparathyroidism spontaneously resolved after FNAB and the parathyroid hormone (PTH) levels normalized after surgery. The first patient maintained a normal level of PTH for 6 years, and the second patient received kidney transplantation 6 years after surgery, and the normal level of PTH was confirmed for 13 years. Caution is needed while performing FNAB for diagnosis of hyperparathyroidism and during decision making regarding whether to observe the patient or perform surgery after spontaneous remission due to bleeding or infarction.
\end{abstract}

Keywords: Hyperparathyroidism; fine-needle aspiration biopsy (FNAB); spontaneous remission; case report

Submitted Jan 05, 2021. Accepted for publication Apr 23, 2021.

doi: $10.21037 / \mathrm{gs}-21-6$

View this article at: https://dx.doi.org/10.21037/gs-21-6

\section{Introduction}

Primary hyperparathyroidism (PHPT) is the third most common endocrine disease, and most patients with PHPT have an adenoma (1). The optimal treatment for PHPT is the surgical removal of the lesion. However, in few published cases, autoinfraction in the lesion led to spontaneous remission of hyperparathyroidism $(2,3)$. In more than half of PHPT cases, permanent improvement has been noted after spontaneous remission; however, in some PHPT cases, that the spontaneous remission was temporary $(4,5)$. There have been few reports of PHPT remission caused by hemorrhage of a parathyroid adenoma after fine needle aspiration biopsy (FNAB).
Moreover, FNAB for diagnostic purposes in patients with hyperparathyroidism is controversial. In two cases presented here, hemorrhage due to the use of FNAB for the diagnosis of hyperparathyroidism led to spontaneous remission a parathyroid adenoma, which was confirmed on preoperative imaging and postoperative histological analysis. We present the following cases in accordance with the CARE reporting checklist (available at https://dx.doi.org/10.21037/gs-21-6).

\section{Case presentation}

Case 1

A 58-year-old man who was undergoing osteoporosis 


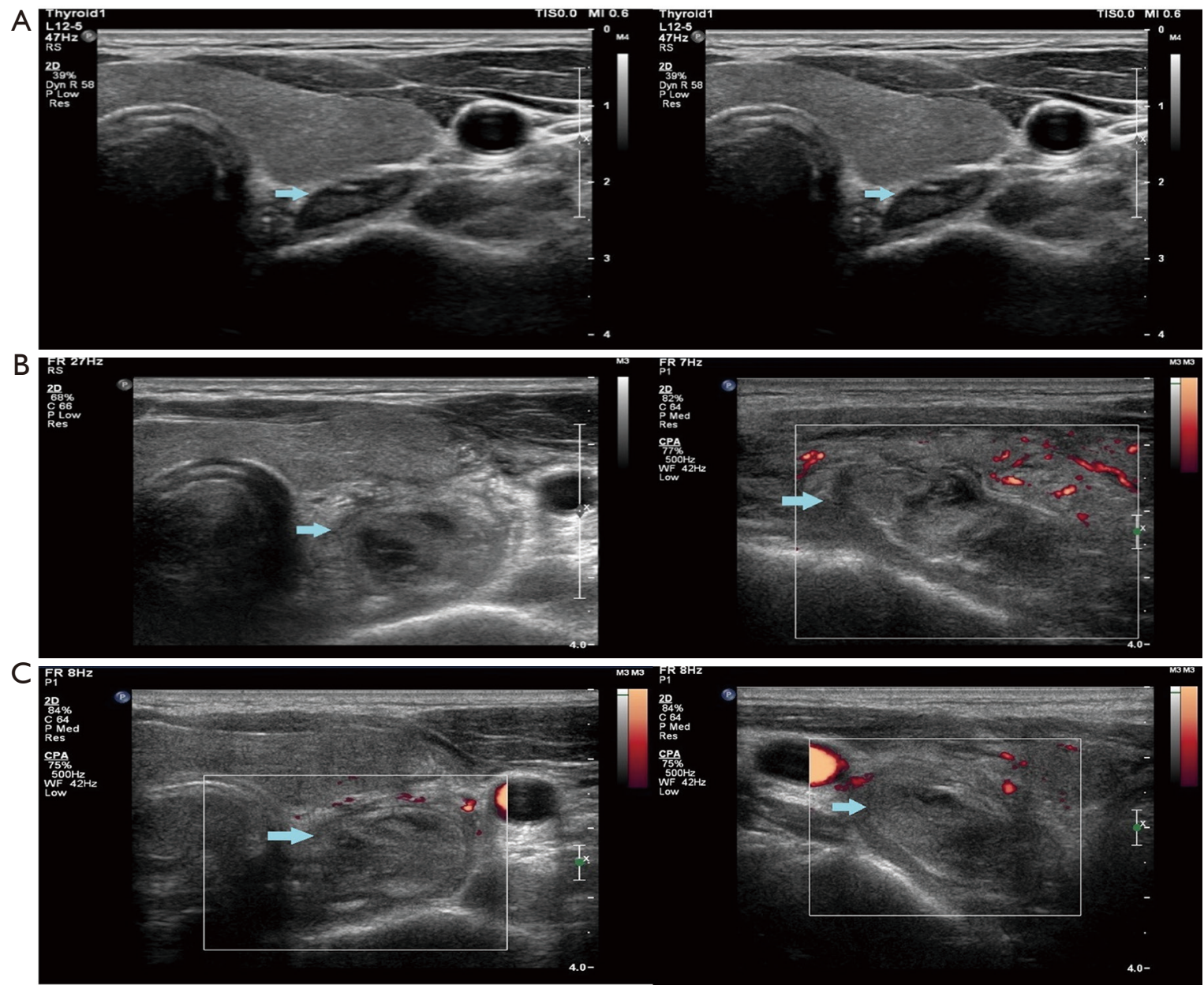

Figure 1 Ultrasound of neck (case 1). (A) An isoechoic mass with well demarcated clear border before FNAB (blue arrow); (B) enlargement of a heterogeneous hyperechoic mass mixed with intracapsular hematoma 4 days after FNAB (blue arrow); the patient had developed swelling and neck pain accompanied by odynophagia; (C) 10 days after the FNAB, enlargement of the heterogeneous hyperechoic mass mixed with intracapsular hematoma remained (blue arrow). FNAB, fine needle aspiration biopsy.

treatment visited Severance Hospital for treatment. During consultation with the Endocrine Department, hypercalcemia and elevated parathyroid hormone (PTH) levels were confirmed [PTH level, $267.3 \mathrm{pg} / \mathrm{mL}$ (normal, $15-65 \mathrm{pg} / \mathrm{mL}$ ); serum calcium level, $11.1 \mathrm{mg} / \mathrm{dL}$ (normal, $8.5-10.5 \mathrm{mg} / \mathrm{dL})]$. Ultrasound evaluation revealed a left extrathyroidal neck mass measuring $2.6 \mathrm{~cm} \times 1.6 \mathrm{~cm}$, which was suspected to be a parathyroid adenoma (Figure $1 A$ ). FNAB of the extrathyroidal mass was performed; the slide was assessed to be nondiagnostic by a pathologist due to blood mixed cytology. There were no immediate complications, and the patient did not complain of any discomfort. Four days after FNAB, the patient developed swelling and neck pain accompanied by odynophagia.
He presented to an outpatient radiology clinic, where ultrasound evaluation revealed enlargement of the original mass $(2.9 \mathrm{~cm} \times 2.1 \mathrm{~cm})$ that was mixed with an intracapsular hematoma in the left neck (Figure 1B,C). Functional singlephoton emission computed tomography using ${ }^{99 \mathrm{~m}} \mathrm{Tc}-$ sestamibi (SPECT MIBI), performed for localization before surgery, revealed a non-uptaken lesion measuring $2.5 \mathrm{~cm}$ in the left neck between the thyroid gland and the esophagus.

The patient underwent left-focused parathyroidectomy. Because there was severe fibrosis and an inflamed mass, it was difficult to confirm the parathyroid lesion; hence, a part of the specimen was sent for pathological examination intraoperatively. Frozen biopsy revealed fibrotic tissue with necrosis. Therefore, the second specimen that 

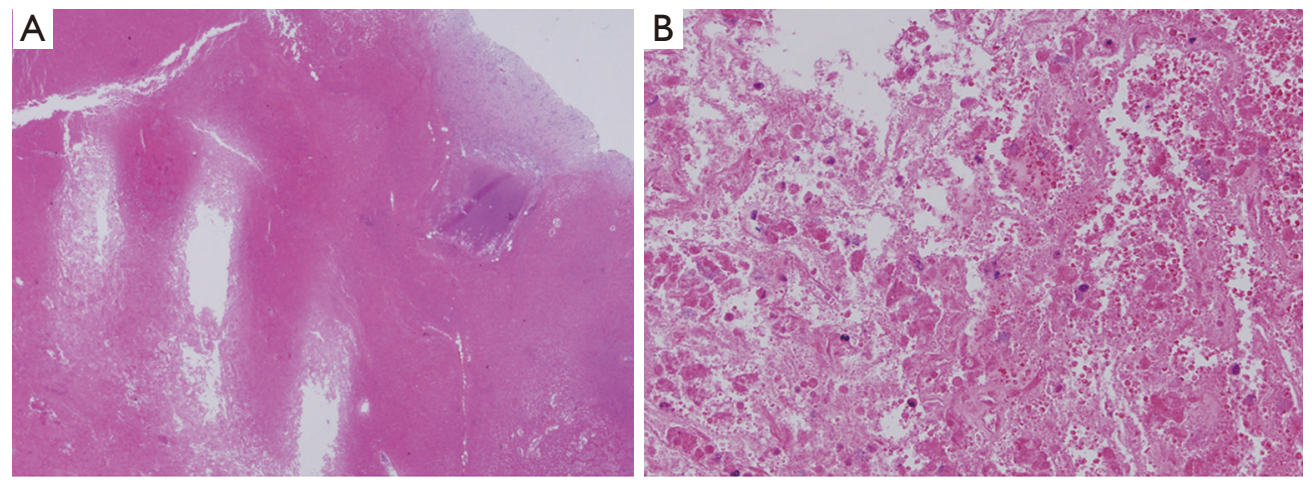

Figure 2 Microscopic appearance of the parathyroid lesion (case 1). (A) Near total necrotic material is seen on hematoxylin-eosin stain ( $\times 40$ ); (B) parathyroid cells confirmed using immunochemical staining for chromogranin A $(\times 200)$.

was excised from mass-like fibrotic tissues was sent for pathological evaluation. The second specimen revealed near total necrotic material. The pathologist did not confirm the specimen to be a parathyroid lesion. After confirming that intraoperative PTH level had dropped from 267 to $69.4 \mathrm{pg} / \mathrm{dL}$, the operation was completed.

A permanent pathology report revealed that the parathyroid specimen was characterized by near total necrotic material with parathyroid gland cells, confirmed using immunohistochemical staining for chromogranin A. There was no focus of parathyroid hyperplasia or adenomatous lesion in the pathological review (Figure 2). Therefore, the suspected parathyroid adenoma was thought to have changed to iatrogenic necrosis due to FNABinduced intracapsular hemorrhage, and only normal parathyroid tissue remained. Preoperative SPECT MIBI and immunohistopathological findings demonstrated the functional change in the parathyroid adenoma due to FNAB-induced intracapsular hemorrhage.

\section{Case 2}

A 37-year-old woman receiving hemodialysis treatment for end-stage renal disease was diagnosed with secondary hyperparathyroidism. Her PTH level was $447.1 \mathrm{pg} / \mathrm{mL}$ (normal, 15-65 $\mathrm{pg} / \mathrm{mL}$ ), and her serum calcium level was $11.1 \mathrm{mg} / \mathrm{dL}$ (normal, $8.5-10.5 \mathrm{mg} / \mathrm{dL}$ ). Functional SPECT MIBI revealed bilateral uptake lesion in the lower thyroid (Figure 3). Ultrasound evaluation revealed a right lower extrathyroidal neck mass measuring $2.0 \mathrm{~cm} \times 1.5 \mathrm{~cm}$ and a left lower extrathyroidal neck mass measuring $1.3 \mathrm{~cm} \times 1.2 \mathrm{~cm}$, which was suspected to be a parathyroid lesion (Figure $4 A, B$ ). One pass was made into the each extrathyroidal mass using a 24-gauge needle, and the slides were identified by a pathologist as chief cell proliferation, favoring parathyroid hyperplasia. The level washout PTH from the extrathyroidal mass was $>1,000 \mathrm{pg} / \mathrm{mL}$. There were no immediate complications, and the patient did not complain of any discomfort. Ten days after FNAB, the patient presented to an outpatient nephrology and endocrinology clinic. Her PTH level had dropped from 447 to $68.3 \mathrm{pg} / \mathrm{mL}$, and her serum calcium level had dropped from 11.1 to $9.7 \mathrm{mg} / \mathrm{dL}$. After consultation with an endocrine surgeon, she decided to undergo parathyroidectomy 2 months later. Because of the decreasing PTH and calcium level, we rechecked the PTH and calcium levels. The serum calcium level remained at $9.7 \mathrm{mg} / \mathrm{dL}$, but the PTH level had slightly increased to $102.2 \mathrm{pg} / \mathrm{mL}$. When the patient was admitted for surgery, routine chemistry laboratory test results, including calcium and electrolyte levels, were rechecked. The serum calcium level was $9.3 \mathrm{mg} / \mathrm{dL}$. The patient underwent bilateral total parathyroidectomy; which appeared normal, was minced to a size of $<1 \mathrm{~mm}$ and auto-transplanted to the brachialis muscle in the right forearm. Frozen biopsy revealed parathyroid hyperplasia. After the operation was completed, we confirmed that the intraoperative PTH level had dropped from 102.2 to $30.5 \mathrm{pg} / \mathrm{dL}$.

In line with our expectations, a permanent pathology report revealed that the specimen obtained during FNAB had central infarction and hemosiderin deposits. There was still a focus of hyperplastic parathyroid lesion in the pathologic review (Figure 5). All information in both cases were obtained through retrospective literature review, and informed consent was not required as it did not contain information that could identify the patients. All procedures performed in studies involving human participants were in 


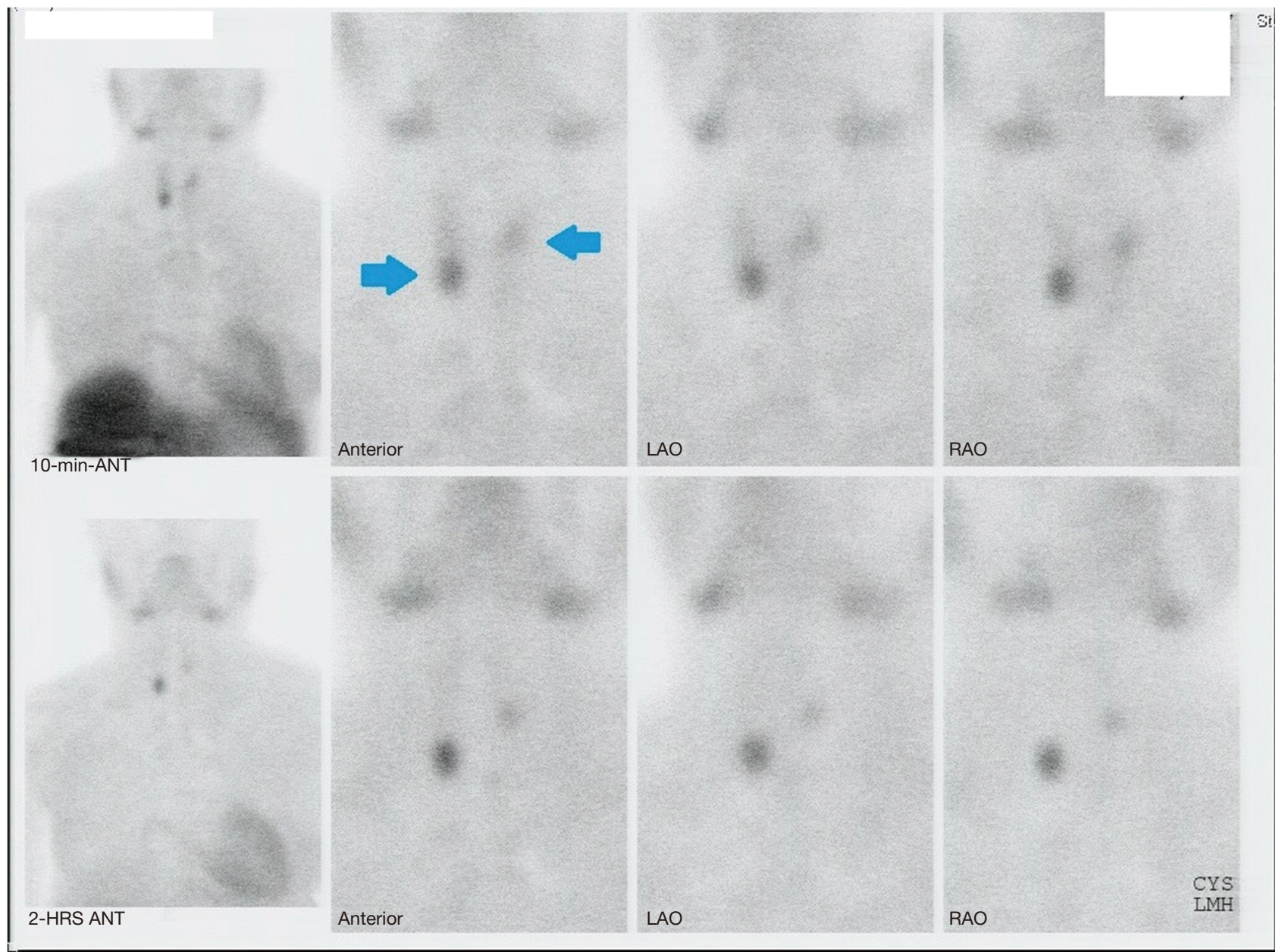

Figure 3 Parathyroid sestamibi scan (case 2). Two foci of increased uptake near the inferior pole of the right thyroid and superior pole of the left thyroid (blue arrows) are seen in the delayed phase.

accordance with the ethical standards of the institutional and/or national research committee(s) and with the Helsinki Declaration (as revised in 2013).

\section{Discussion}

Spontaneous remission of PHPT is uncommon; however, in most cases, it can lead to the resolution of the disease. Spontaneous remission of PHPT is usually asymptomatic and can sometimes be recognized because the patient's serum calcium levels spontaneously decrease. In several reports, spontaneous remission of PHPT was accompanied by sudden discomfort and pain in the neck $(3,6,7)$.

Nylen et al. have proposed that spontaneous remission of PHPT can be divided into three categories by the degree of hemorrhage-autoinfarction (necrosis without hemorrhage), acute extracapsular hemorrhage, or acute intracapsular hemorrhage (8). Wootten et al. summarized these three categories and performed an analysis of approximately 50 cases of spontaneous remission of PHPT (4). These three categories were seen in a similar number of cases, led to similar serum calcium levels after spontaneous remission, and had a similar distribution across age and sex. Acute clinical abnormalities after acute intracapsular hemorrhage were less common and serum calcium levels prior to spontaneous remission of PHPT were higher than others. In contrast, acute extracapsular hemorrhage was related to more serious clinical symptoms such as hoarseness, ecchymosis, and subcutaneous hematoma (4).

In the first case, intracapsular hemorrhage was confirmed in the final pathology report, but the clinical manifestations were somewhat severe including neck pain and severe 


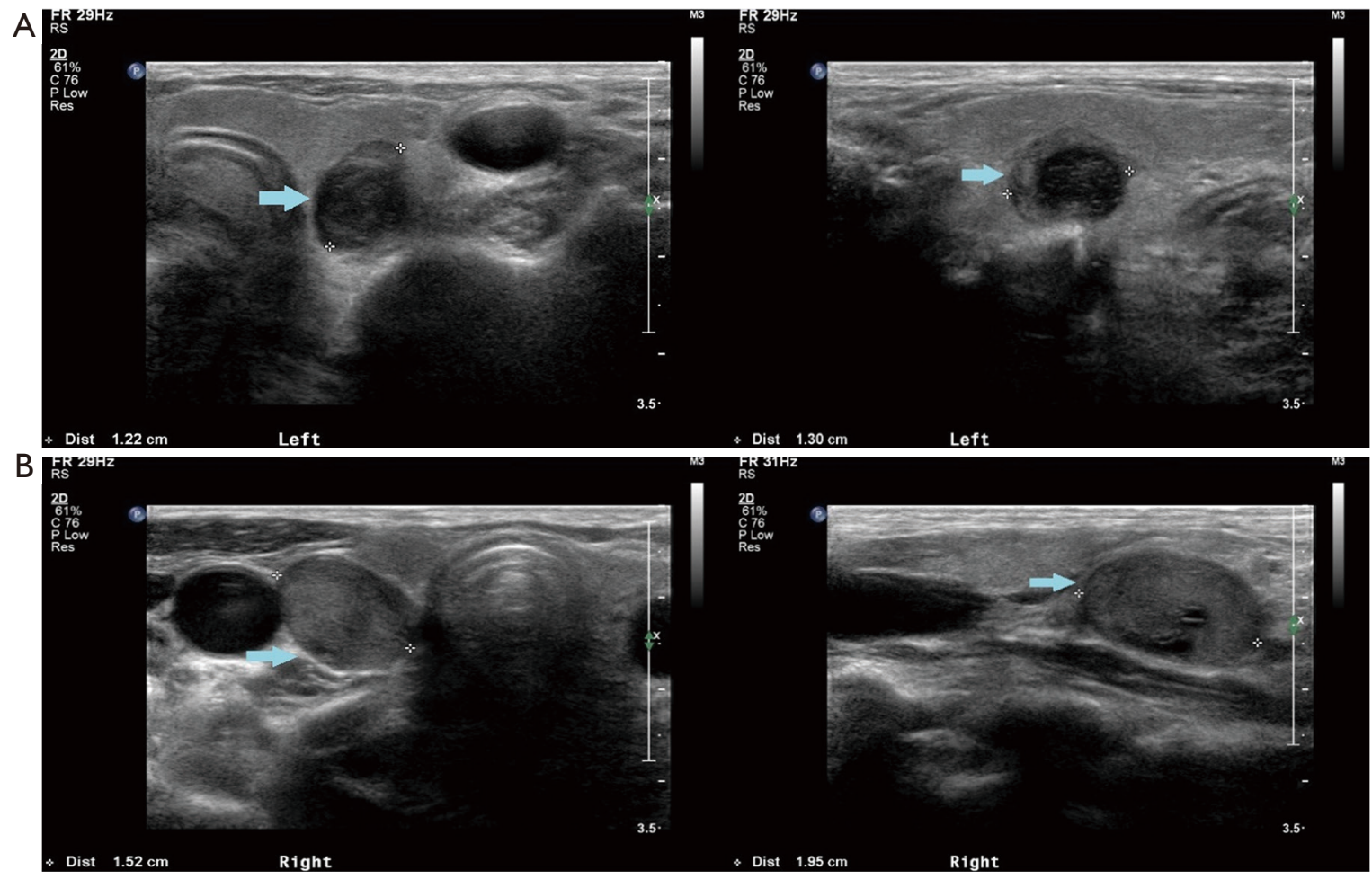

Figure 4 Ultrasound of the neck (case 2). (A) A nodule without a clear border mimicking a thyroid nodule in the left thyroid (blue arrow); (B) A nodule with clear and well-demarcated border in the posteroinferior right thyroid (blue arrow).
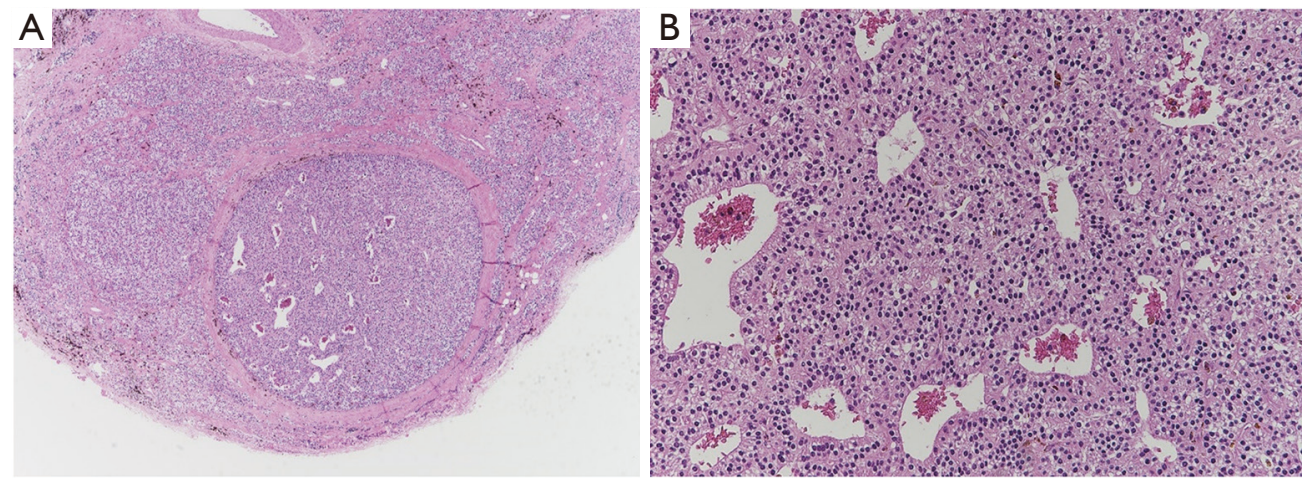

Figure 5 Microscopic appearance of the parathyroid lesion (case 2). (A) Parathyroid hyperplasia with central infarction and hemosiderin deposits (hematoxylin-eosin stain, $\times 40$ ); (B) Parathyroid cells confirmed using immunochemical staining for chromogranin A $(\times 200)$.

swelling. The second case did not involve any notable clinical symptoms.

Some authors have reported that spontaneous resolution is temporary; therefore, close follow-up or parathyroidectomy is required $(3,7,9)$. However, other authors have reported that cyclical secretion of PTH by the parathyroid adenoma causes hypercalcemic crises, followed by a spontaneous decrease in
PTH levels. However, the patient had renal insufficiency; hence, the cyclic PTH fluctuation may have been caused by pending chronic renal failure (10). In cases reported by Kwak et al., the PTH level rebounded after 6 months; hence, surgical treatment was provided; the parathyroid lesion in the permanent biopsy sample was confirmed to be a parathyroid adenoma (11). 
In the first case in our report, iatrogenic hemorrhage due to the use of FNAB led to unexpected remission of the parathyroid adenoma. We worried about airway compression due to hemorrhage and performed serial neck ultrasonography. Although the SPECT MIBI results were negative, the parathyroidectomy was performed according to schedule. We could not identify parathyroid cells in the frozen biopsy, which revealed only fibrotic tissue with necrosis. The spontaneous remission of hyperparathyroidism may have been caused by intracapsular hemorrhage. In the second case in our report, based on serial laboratory follow-up tests, we could prove that the PTH and serum calcium levels decreased to the normal range. Both cases in our report remained in that state for a long time after remission. The first patient maintained a normal level of PTH for 6 years, and the second patient received kidney transplantation 6 years after surgery, and the normal level of PTH was confirmed for 13 years.

FNAB of the parathyroid gland has not been generally recommended and its use is controversial. Some studies have reported that FNAB with PTH testing is helpful in diagnosing parathyroid disease. Abraham et al. found that the sensitivity of cytological evaluation was $91 \%$ with a specificity of $95 \%$ (12). Erbil et al. reported that the sensitivity and positive predictive value of PTH testing with FNAB to localize parathyroid adenoma were $100 \%$ (13).

Recently, a case report described a case of acute airway comprise due to spontaneous hemorrhage of a parathyroid adenoma (14). The parathyroid gland is an organ vulnerable to ischemia caused by rapid growth. Studies on spontaneous and iatrogenic remission support that procedures, such as percutaneous ethanol injection for hyperparathyroidism, may be performed in severely sick patients who cannot undergo general anesthesia (15). However, the potential for iatrogenic airway damage due to bleeding caused by the injection procedure should always be kept in mind.

\section{Conclusions}

Spontaneous remission of hyperparathyroidism is a rare phenomenon, and disease-causing evidence has recently emerged and explained. It might be possible to predict whether permanent or temporary remission of hyperparathyroidism depends on the degree of abnormal change caused by bleeding. However, it is unclear why spontaneous remission is maintained or leads to disease recurrence. In similar cases, more attention should be given to patient's quality of life. Further studies are needed to confirm the risk or justification of FNAB for parathyroid adenoma and understand whether it is a more reasonable management strategy than close follow-up or immediate surgery.

\section{Acknowledgments}

Funding: None.

\section{Footnote}

Reporting Checklist: The authors have completed the CARE reporting checklist. Available at https://dx.doi. org/10.21037/gs-21-6

Conflicts of Interest: All authors have completed the ICMJE uniform disclosure form (available at https://dx.doi. org/10.21037/gs-21-6). The authors have no conflicts of interest to declare.

Ethical Statement: The authors are accountable for all aspects of the work in ensuring that questions related to the accuracy or integrity of any part of the work are appropriately investigated and resolved. All information in both cases were obtained through retrospective literature review, and informed consent was not required as it did not contain information that could identify the patients. All procedures performed in studies involving human participants were in accordance with the ethical standards of the institutional and/or national research committee(s) and with the Helsinki Declaration (as revised in 2013).

Open Access Statement: This is an Open Access article distributed in accordance with the Creative Commons Attribution-NonCommercial-NoDerivs 4.0 International License (CC BY-NC-ND 4.0), which permits the noncommercial replication and distribution of the article with the strict proviso that no changes or edits are made and the original work is properly cited (including links to both the formal publication through the relevant DOI and the license). See: https://creativecommons.org/licenses/by-nc-nd/4.0/.

\section{References}

1. Adler JT, Sippel RS, Chen H. New trends in parathyroid surgery. Curr Probl Surg 2010;47:958-1017.

2. Cetani F, Ambrogini E, Faviana P, et al. Spontaneous short-term remission of primary hyperparathyroidism 
from infarction of a parathyroid adenoma. J Endocrinol Invest 2004;27:687-90.

3. Pereira FA, Brandao DF, Elias J, et al. Parathyroid adenoma apoplexy as a temporary solution of primary hyperparathyroidism: a case report. J Med Case Rep 2007;1:139.

4. Wootten CT, Orzeck EA. Spontaneous remission of primary hyperparathyroidism: a case report and metaanalysis of the literature. Head Neck 2006;28:81-8.

5. Novodvorsky P, Hussein Z, Arshad MF, et al. Two cases of spontaneous remission of primary hyperparathyroidism due to auto-infarction: different management and their outcomes. Endocrinol Diabetes Metab Case Rep 2019. doi: 10.1530/EDM-18-0136.

6. Micale SJ, Kane MP, Busch RS. Spontaneous resolution of primary hyperparathyroidism in parathyroid adenoma. Case Rep Endocrinol 2012;2012:793753.

7. Kataoka K, Taguchi M, Takeshita A, et al. Recurrence of primary hyperparathyroidism following spontaneous remission with intracapsular hemorrhage of a parathyroid adenoma. J Bone Miner Metab 2008;26:295-7.

8. Nylen E, Shah A, Hall J. Spontaneous remission of primary hyperparathyroidism from parathyroid apoplexy. J Clin Endocrinol Metab 1996;81:1326-8.

9. Lucas DG, Jr., Lockett MA, Cole DJ. Spontaneous infarction of a parathyroid adenoma: two case reports and

Cite this article as: Ho J, Kim D, Lee JE, Choi S, Choi H, Choi S, Kim J, Kang SW, Lee J, Jeong JJ, Nam KH, Chung WY. Unexpected remission of hyperparathyroidism caused by hemorrhage due to the use of fine-needle aspiration biopsy: two cases report. Gland Surg 2021;10(6):2047-2053. doi: 10.21037/gs21-6 review of the literature. Am Surg 2002;68:173-6.

10. Makita N, Iiri T, Sato J, et al. An instructive case suggesting cyclical primary hyperparathyroidism. Endocr J 2006;53:311-6.

11. Kwak JY, Kim EK, Moon HJ, et al. Parathyroid incidentalomas detected on routine ultrasound-directed fine-needle aspiration biopsy in patients referred for thyroid nodules and the role of parathyroid hormone analysis in the samples. Thyroid 2009;19:743-8.

12. Abraham D, Sharma PK, Bentz J, et al. Utility of ultrasound-guided fine-needle aspiration of parathyroid adenomas for localization before minimally invasive parathyroidectomy. Endocr Pract 2007;13:333-7.

13. Erbil Y, Salmaslioglu A, Kabul E, et al. Use of preoperative parathyroid fine-needle aspiration and parathormone assay in the primary hyperparathyroidism with concomitant thyroid nodules. Am J Surg 2007;193:665-71.

14. Garrahy A, Hogan D, O'Neill JP, et al. Acute airway compromise due to parathyroid tumour apoplexy: an exceptionally rare and potentially life-threatening presentation. BMC Endocr Disord 2017;17:35.

15. Tominaga Y, Kakuta T, Yasunaga C, et al. Evaluation of Parathyroidectomy for Secondary and Tertiary Hyperparathyroidism by the Parathyroid Surgeons' Society of Japan. Ther Apher Dial 2016;20:6-11. 